\title{
The function of stimulus predifferentiation pretraining in complex problem-solving
}

\author{
MARVIN L. SCHROTH \\ UNIVERSITY OF SANTA CLARA
}

The present experiment was designed to determine the effects of various types of stimulus predifferentiation pretraining on complex problem-solving and, in addition, the role of task complexity in transfer. Ss were 150 second grade children assigned to six groups, including five treatment conditions, and matched for sex. Directed Attention pretraining proved to facilitate the greatest amount of transfer in solving the oddity problem, and transfer was greater from the difficult task to the easy task than in the reverse situation.

Stimulus predifferentiation pretraining as a variable facilitating transfer has been extensively investigated in learning tasks involving simple $S-R$ components. The typical procedure in these studies has been to provide the subject with some type of stimulus pretraining in which the subject is given an opportunity to differentiate among a number of stimulus items. Such stimuli are then used in a transfer task, typically of a motor variety.

The primary purpose of the present study is to further explore the role of stimulus predifferentiation pretraining as it relates to transfer in complex problem-solving. In addition, the variable of task complexity in transfer was examined; some experiments report transfer from an easy to a hard one is greater while other studies demonstrate the converse is sometimes true (Deese, 1958).

Attainment of these objectives was carried out by using five specific pretraining methods, the first two of which also represent the task complexity variable, as a prelude to solving oddity problems. These are complex problems, described by Harlow (1942), which provide two or more cues on individual trials.

Method

Subjects. Ss were 150 second grade children. They were assigned to six groups and matched for sex so that each group was composed of 15 boys and 10 girls.

Stimuli. The method used to construct the stimuli used in this experiment is described in the following paragraphs.

The first set of stimuli consisted of "metric" shapes which were constructed by a technique developed by Fitts, Weinstein, Rappaport, Anderson, \& Leonard (1956). After two shapes were selected at random, there followed a systematic derivation of two "families" of shapes from the initial prototypes.

The last step consisted of drawing the metric figures in black ink on 127 in. $\times 14$ in. pieces of cardboard. All three figures on a given card were the same size (4 in. $x 4$ in.), but two (derived from the first proto- type form) were identical, while the third (derived from the second prototype form) was different. These figures were so arranged that the odd shaped stimulus appeared in each of the three possible positions four times out of the set of 12 .

The same method utilized in the construction of the first set of stimuli was used for the second set except for the selection of two new prototype forms. Following the derivation of the two new families of figures, three colors (red, blue, and yellow) were selected so as to increase the complexity of the problems through the addition of another stimulus dimension, color.

Construction of the third set of stimuli was conducted in the same fashion as in the preceeding sets except for the selection of two new prototypes. In addition to the cues of shape and color, these figures were made still more complex by varying the sizes of all three figures on any given card. The sizes selected were: (1) 4 in. $x 4$ in., (2) 2.6 in $\times 2.6$ in., and (3) 1.9 in. $\times 1.9$ in.

Feedback or knowledge of results was provided on each trial by a poker chip which was placed immediately behind the correct figure (Ss in some experimental conditions were required to designate their choice of figure by pointing

Design. The initial phase consisted of five specific pretraining methods, based upon Arnoult's (1957) classification, the first two of which also represent the task complexity variable. Groups 1 (RSR, E) and 2 (RSR, D) typify the Relevant S-R pretraining method or "classical transfer design," in which the responses to Task $A$ bear a significant relationship to the responses made to Task B. In the present case, the responses (oddity) to the pretraining and transfer tasks were identical. Groups 3,4 and 5 represent the stimulus predifferentiation pretraining conditions of Relevant S (RS). Directed Attention (DA) and Irrelevant S (IS) respectively; Group 6 (CG) served as a control.

Group 1. Ss of this group were given practice in solving oddity problems using Set 1 class of stimuli. Under this condition the correct and reinforced response was always to the stimulus figure odd in shape. This represents the easy-difficult experimental condition, using Relevant S-R pretraining.

Group 2. Ss of this group were given practice in solving oddity problems using set 3 class of stimuli. The reinforced response was always to the stimulus figure odd in shape. This represents the difficult-easy experimental condition using the Relevant S-R method. 
Group 3. This group was administered Set 2 class of stimuli. Under this condition, the reinforced response was always to the figure occupying the middle position in the set of three. For the purposes of this study the positional response of "middleness" was considered as unrelated to the "oddity" response, constituting the Relevant S pretraining method.

Group 4. This group was administered Set 2 class of stimuli, but was not required to make any type of overt response to the stimulus cords. Instead the Ss were required by instructions to notice how the figures differed in shape and color, representing the Directed Attention pretraining method. Amount of time in practice was equated with the other experimental groups.

Group 5. Ss in this group were administered the House-Tree-Person (HTP) projective test, in which amount of time was equated with Groups $1-4$. This task bears no relationship to the transfer task, establishing the Irrelevant $S$ pretraining method. This experimental situation is often used to evaluate the effects of warmup.

Group 6. Ss in this group were given no pretraining prior to the undertaking of the transfer task and served as the control group.

Following the various pretraining conditions, all Ss were administered the oddity problem using Set 2 class of stimuli. Both the pretraining and transfer portions of the experiment consisted of 30 trials.

\section{Results and Discussion}

To test for transfer, the performances of the experimental groups on Task B were compared with the control group and one another.

The mean number of correct responses over the 30 testing trials was analyzed by simple analysis of variance. The resulting $F$ of 5.11 is significant at the .01 level of confidence, using a two-tailed test $(\mathrm{df}=5 / 144)$.

To determine which means differed significantly from one another, the Newman-Keuls method for multiple comparisons was employed. Group 4 (DA) differed significantly from all other groups while Group 2 (RSR, D) differed significantly from Groups 1 (RSR, E), 5 (IS), and 6 (CG) at the .05 level of confidence.

The most significant finding in this experiment centered around the fact that Directed Attention method of pretraining, Group 4 (DA), proved to facilitate a greater amount of transfer than any other pretraining method, including Relevant S-R, Group 2 (RSR, D), which also resulted in positive transfer. The pretraining methods provided Groups 3 (RS), 1 (RSR, E), and 5 (IS) did not facilitate transfer. These findings will be discussed below in terms of a mediating processes theory.

There exist theoretical grounds for supposing that similar mediating processes were involved in the pretraining and transfer tasks for Groups 1-4 and, consequently, one might logically expect these groups to demonstrate positive transfer. For example, both tasks required discrimination, comparison and categorizing of the stimuli. In order to render a correct response on a given trial in either task, it was requisite that Ss possess the capacity to recognize similarities and differences between the stimuli.

It is suggested by the data that Ss best learned to discriminate and categorize the complex stimuli under the Directed Attention condition, Group 4 (DA), in which the various dimensions (color, shape) were pointed out to them. Apparently the other stimulus predifferentiation techniques are insufficient, with the possible exception of Relevant S-R, when complex problems are involved and the pretraining experiences do not carry over to mediate between stimuli and responses on Task $B$.

A second finding in the current study was that pretraining involving a difficult task, Group 2 (RSR, D), facilitated more transfer than pretraining using an easy task, Group 1 (RSR, E). In analyzing the particular situation in the current experiment, the harder task included all of the components of the easy task and permitted additional or greater practice. The easy task had only some of the same stimulus components as the difficult task. These data are in agreement with Deese's statement, "in tasks in which more difficult versions contain all of the stimulus-response elements in simpler versions plus additional ones, transfer may be large" (1958, p. 230).

\section{References}

ARNOULT, M. D. Stimulus predifferentiation: Some generalizations and hypotheses. Psychol. Bull., 1957, 54, 339-350.

CANTER, G. N. Effects of three types of pretraining on discrimination learning in preschool children. J. exp. Psychol., 1955, 49, 339-342. DEESE, J. The psychology of learning. New York: McGraw-Hill, 1958. FITTS, P. M., WEINSTEIN, M., RAPPAPORT, M., ANDERSON, N., \& LEONARD, A. J. Stimulus correlates of visual pattern recognition. J. exp. Psychol., 1956, 51, 1-11.

GAGNE, R. M., \& BAKER, K. E. Stimulus predifferentiation as a factor in transfer of training. J. exp. Psychol., 1950, 40, 61-73.

HARLOW, H. F. Responses by rhesus monkeys to stimuli having multiple sign values. In Q. McNemar and M. H. Merrill (Eds.), Studies in personality. New York: McGraw-Hill, 1942. Pp. 105-123.

Note

1. The manuscript is based, in part, on a paper presented at the Western Psychological Association convention, San Francisco, 1967. 